\title{
The BrainMap strategy for standardization, sharing, and meta-analysis of neuroimaging data
}

\author{
Angela R Laird ${ }^{1 *}$, Simon B Eickhoff ${ }^{2,3}$, P Mickle Fox', Angela M Uecker ${ }^{1}$, Kimberly L Ray ${ }^{1}$, Juan J Saenz Jr 1,4, \\ D Reese McKay ${ }^{1}$, Danilo Bzdok ${ }^{2,3}$, Robert W Laird ${ }^{4}$, Jennifer L Robinson ${ }^{5,6}$, Jessica A Turner ${ }^{7}$, Peter E Turkeltaub ${ }^{8}$, \\ Jack L Lancaster ${ }^{1}$ and Peter T Fox ${ }^{1}$
}

\begin{abstract}
Background: Neuroimaging researchers have developed rigorous community data and metadata standards that encourage meta-analysis as a method for establishing robust and meaningful convergence of knowledge of human brain structure and function. Capitalizing on these standards, the BrainMap project offers databases, software applications, and other associated tools for supporting and promoting quantitative coordinate-based meta-analysis of the structural and functional neuroimaging literature.

Findings: In this report, we describe recent technical updates to the project and provide an educational description for performing meta-analyses in the BrainMap environment.

Conclusions: The BrainMap project will continue to evolve in response to the meta-analytic needs of biomedical researchers in the structural and functional neuroimaging communities. Future work on the BrainMap project regarding software and hardware advances are also discussed.
\end{abstract}

Keywords: functional neuroimaging, structural neuroimaging, meta-analysis, BrainMap, neuroinformatics, activation likelihood estimation, ALE

\section{Background}

A recent and timely editorial in BMC Research Notes called for a series of educational articles that promote best practices in data sharing in the biomedical sciences [1]. In the domain of neuroimaging research, data sharing is critical for establishing the robust and meaningful convergence of knowledge of human brain function and structure. The need for such data pooling is primarily dictated by the inherent limitations of neuroimaging data. Most important among those are the rather small sample sizes investigated, the low reliability of indirect signals, and the inherent subtraction logic that is only sensitive to differences between two specific conditions.

Progress towards open sharing of reusable original data has been slow, limited by complex data acquisition and analysis techniques that require extensive curation, the size of the data sets, patient confidentiality, as well as a

\footnotetext{
* Correspondence: lairda@uthscsa.edu

'Research Imaging Institute, University of Texas Health Science Center, San Antonio, TX, USA

Full list of author information is available at the end of the article
}

desire on the investigators' part to protect their costly investment and maintain future rights to their data. Nevertheless, several recent efforts have begun to promote neuroimaging data sharing on a large scale, such as the Biomedical Informatics Research Network [2,3], XNAT Central $[4,5]$, the Alzheimer's Disease Neuroimaging Initiative [6,7], and the Human Connectome Project $[8,9]$. These projects focus on sharing complete imaging data sets at the subject level across a wide range of modalities, such as task-based functional magnetic resonance imaging (fMRI), resting state fMRI, structural MRI, diffusion imaging, positron emission tomography, magnetoencephalography, and electroencephalography. These multivariate neuroimaging data can be processed and analyzed in a huge variety of ways using algorithms that are in a continual state of evolution and improvement. As a result, understanding complete data and processing provenance [10] across these diverse data sets remains a significant neuroinformatics challenge for the imaging community.

In contrast to these large-scale, multi-institutional sharing initiatives, the BrainMap project was created as
C Biomed Central

(c) 2011 Laird et al; licensee BioMed Central Ltd. This is an open access article distributed under the terms of the Creative Commons Attribution License (http://creativecommons.org/licenses/by/2.0), which permits unrestricted use, distribution, and reproduction in any medium, provided the original work is properly cited. 
an alternative to sharing raw biomedical images. Instead, BrainMap offers a venue for sharing neuroimaging data in a reduced format as a means to encourage and facilitate the identification of consistent findings on brain activity and structure across multiple data sets [11-14]. Here, we describe the rigorous community standards developed since the inception of functional and structural neuroimaging research that have laid the foundation for the advancement of formal meta-analysis methods. These meta-analyses do not require access to raw image data, but can be achieved via information reported by authors in the published literature. In this report, we address the data and metadata standards that enable neuroimaging meta-analyses and the strategy developed by the BrainMap project to encourage data reuse and sharing throughout the community.

\section{Neuroimaging Data Analysis and Reporting Standards}

Spatial normalization algorithms have been developed and implemented in all of the major neuroimaging software packages (e.g., FSL [15], SPM [16], AFNI [17], etc.) to ensure that data from individual subjects are spatially normalized from a subject's "native" brain space to a "standard" brain space. This data standardization removes the effects of intersubject anatomical variability due to differences in brain size and shape, allowing investigators to report their research findings in a manner that facilitates the comparison and synthesis of results across multiple studies [18]. The location of brain imaging results are hence generally published as three-dimensional coordinates $(x, y, z)$ of the centers of mass of clusters or local maxima of brain activation or structural findings, to provide readers with quantitative summaries of the statistical parametric images, with corresponding $z$ or $t$ statistic values to indicate the strength of the observations. Most commonly, these tables of coordinates refer to locations in Talairach [19] or MNI standard spaces [20]. To facilitate meta-analysis of structural or functional brain findings, it is critical that authors clearly report which standard space was utilized in their publications, as well as which software application was used for spatial normalization, since different applications can yield different results [21]. Frequently, this data description can be incomplete or even inaccurate in the literature, especially when the authors have employed a coordinate conversion algorithm to convert MNI coordinates to Talairach space (or vice versa) [21,22] and do not properly indicate this data transformation. Incomplete data descriptions can have a significant effect on meta-analysis outcomes [23], and can be remedied by stronger adherence to the data reporting standards set forth by the fMRI Methods Working Group [24].

Capitalizing on these community standards for data analysis and reporting, the BrainMap project was conceived in
1988 and originally developed as a web-based interface to guide users through search, retrieval, and visualization of a coordinate-based database of functional neuroimaging results [25]. After more than 20 years of development, BrainMap has evolved into a much broader project whose software and data have been utilized in nearly 130 publications, with half of those articles published in the last two years [26]. In contrast to other neuroimaging databases, BrainMap provides not only data for meta-analyses and data mining, but also distributes software and concepts for quantitative integration of data. Currently, the BrainMap project includes two neuroimaging databases, three desktop software applications, one web-based application, and several other tools that serve ancillary functions for carrying out meta-analyses. Below we describe recent updates to the project and provide an educational description for performing meta-analyses in the BrainMap environment (an overview is depicted in Figure 1); this information reflects the new software versions that were released in August 2011.

\section{Findings \\ BrainMap Databases}

BrainMap was initially developed as a database for functional neuroimaging studies reporting brain activation patterns as tabular-formatted three-dimensional stereotactic coordinates. Metadata describing the experimental design and data processing pipeline for each study are manually extracted from each publication, along with the coordinates, by internal BrainMap staff members or external users from the community. Generally, the latter is supported by investigators who are interested in performing a meta-analysis on a given topic and consequently wish to increase BrainMap's volume of studies relevant to this topic. Together, the extracted metadata and coordinate data for each publication comprise the database content. Currently, BrainMap archives the results of 2,114 functional neuroimaging publications, which include 39,672 subjects and report 79,577 activation locations across 9,994 experiments. This volume is estimated to include approximately $20 \%$ of the relevant literature [27].

In 2007, the BrainMap team initiated efforts to expand into archiving structural neuroimaging data. Specifically, voxel-based morphometry (VBM), which is a statistical analysis for investigating structural differences between two groups of subjects (e.g., areas of increased gray matter density for patients vs. healthy controls), also had achieved community standardization such that results reported in the form of stereotactic coordinates had become the norm in the same form as for functional neuroimaging data. Formal integration by meta-analyses on structural neuroimaging findings has thus become possible [28-30]. Following multiple years of data entry, as well as database, servlet, and software programming and development, the 


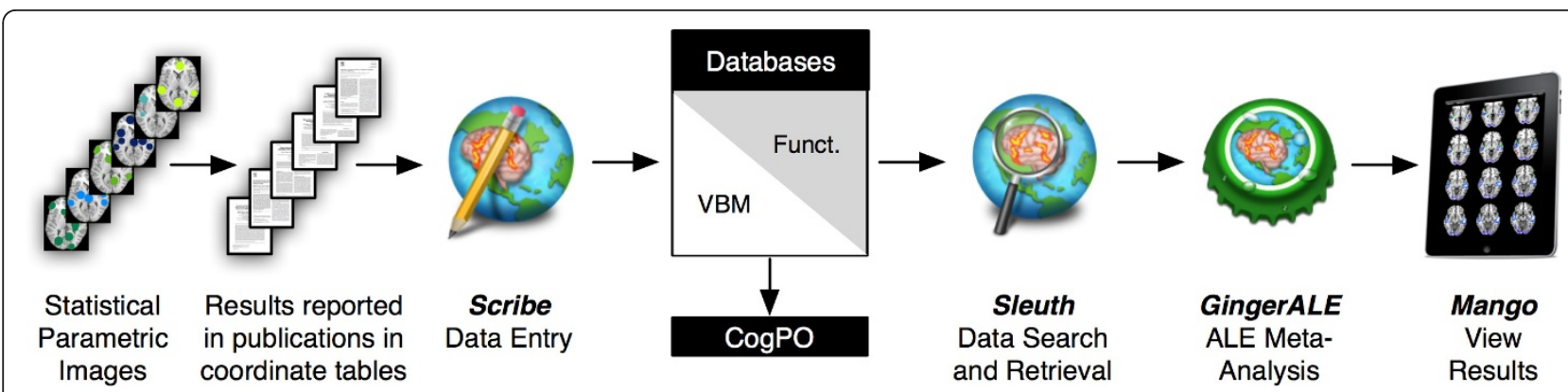

Figure 1 The BrainMap Procedure for Coordinate-Based Meta-Analyses. In the human neuroimaging literature, investigators frequently compute a series of statistical parametric images that summarize the group results observed in their functional or voxel-based morphometry neuroimaging experiments. From these images, the coordinates $(x, y, z)$ of the activation clusters (or clusters of structural differences) are extracted and published in tabular format. Scribe is used to input these coordinates and the associated metadata for these experiments into the BrainMap functional or VBM databases. Once the entries are inserted into the appropriate database, Sleuth is used to search and retrieve coordinates and metadata, and filter the search results to create a data set suitable for meta-analysis. GingerALE is used to perform activation likelihood estimation (ALE) meta-analysis of the data, and these results can be viewed in Mango, or any similar image viewer. As an ancillary tool, the Cognitive Paradigm Ontology (CogPO) has been developed from the BrainMap schema for describing cognitive neuroimaging experiments, and can be used by any researcher to aid in the annotation and formal representation of their own experiments [52].

BrainMap VBM database has been released to the public and is now live. Currently, BrainMap VBM archives the results of 729 voxel-based morphometry publications, which includes 50,375 subjects reporting 15,206 locations of structural differences across 2,231 experiments. While the BrainMap VBM database is much smaller than the functional database, this volume of the literature is also much smaller (939 total eligible publications), and it is hence estimated that the database includes approximately $78 \%$ of the eligible VBM studies.

\section{Database Infrastructure}

Both the BrainMap functional and VBM databases are managed with Oracle, a commercial relational database management system [31]. BrainMap's data resides on a Sun Microsystem workstation running Oracle Solaris 10 at the Research Imaging Institute in San Antonio, TX. The Oracle Corporation's Object-Relational Database Management System was utilized when designing BrainMap's database structure. Object-relational databases have a high-level structure that allows for defining data as objects instead of a collection of items in tables. Treating objects as cohesive units simplifies storing, updating, and retrieving data, as well as defining relationships between objects. The ability to quickly fetch object data is extremely helpful when communicating with BrainMap's Object-Oriented client programs.

\section{BrainMap Software Applications}

BrainMap's client programs are written in the Java programming language that may run under PC, Macintosh, and UNIX operating systems. The use of Java makes updating and distributing these applications simpler for both developers and users. There are three main desktop applications that provide access to the BrainMap databases: Scribe, Sleuth, and GingerALE.

1. Scribe allows users to input data and metadata from publications into the databases using the BrainMap taxonomy [32]. In previous versions, Scribe only provided access to functional submissions; however, the newly released version 2.0 allows users to create entries for either the functional or VBM databases. When Scribe is launched, a dialog window asks users to select which type of paper they wish to code, functional or VBM. Following this, the main application window is configured to match the user's selection. Functional submissions are created as .ent files while the VBM database archives .vbm files, which allows each type of submission to be easily identified. In addition to integrating the interface for functional and VBM submissions, we have also improved how the application functions when there is no active internet connection, as this caused some problems in previous software versions.

2. Sleuth allows users to search and retrieve coordinate data and metadata from the databases. A radio button gives users the option of searching either the functional or VBM database; simultaneous searching of both databases is not permitted to avoid a conflation of both types of imaging results. The graphical user interface of Sleuth has been redesigned in version 2.0, and now allows users to build searches with multiple criteria using an interface that was inspired by the playlist building feature in Apple's iTunes software [33]. In addition, searches are now more rapidly executed as a result of server side optimizations to the database architecture in which search results are 
pre-generated instead of being generated dynamically each time a search is made. Once a search is executed, users are able to examine and filter the query results in workspace panel. As part of this process, the software also allows the brain-based visualization of results with individual experiments being toggled on and off. Most recently, Sleuth now offers the ability to search for studies identifying functional or structural results located in a three-dimensional arbitrary-shaped region of interest (ROI) in Talairach or MNI space. To carry out these image-based ROI searches, all user-originated files must conform to a strict format: ROIs must be formatted as binary NIfTI [34] images with $1 \times 1 \times 1$ $\mathrm{mm}^{3}$ resolution, and the ROI must not extend across more than 500 voxels. These stringent requirements are enforced to ensure a timely response from the database; more advanced hardware solutions are currently being evaluated to reduce these technical limitations and allow rapid image-based ROI searches of greater volume. Other Sleuth tools include the ability to: (1) generate a histogram of metadata results that describe the paradigms and behavioral domains associated with experiments in the current workspace, and (2) export workspaces in multiple formats, including images in NIfTI format (nii), EndNote [35] citation files (txt), or files suitable for meta-analysis using the GingerALE application (txt). Meta-analysis coordinate files can be exported in the form of either Talairach or MNI coordinates.

3. Ginger $\boldsymbol{A} \boldsymbol{L} \boldsymbol{E}$ allows users to carry out activation likelihood estimation (ALE) meta-analyses using BrainMap-formatted coordinate-based data in Talairach or MNI space. In ALE, a set of coordinates retrieved via Sleuth, which are identified by the user as suitable for meta-analysis, are input to GingerALE, blurred with a Gaussian distribution to accommodate the associated spatial uncertainty, and a statistical parameter is computed that estimates convergence across the modeled brain images and measures the likelihood of activation at each voxel in the brain. ALE was originally developed by Turkeltaub et al. [36], but the algorithm has undergone several revisions since then. When initially integrated into the BrainMap environment, a statistical framework was developed for multiple comparisons corrections and allowing two sets of coordinates to be contrasted [37]. In 2009, the algorithm was extensively modified to: (1) model the spatial uncertainty of each brain location using an estimation of the intersubject and interlaboratory variability typically observed in neuroimaging experiments, and (2) calculate the above-change clustering between experiments (i.e., random-effects analysis), rather than between foci (i.e., fixed-effects analysis) [38]. Most recently, we published a modification of the ALE algorithm that minimizes both within-experiment and within-group effects, further optimizing the ALE technique [39]. These algorithms are available in the newest software release, GingerALE version 2.1. GingerALE 2.1 also includes a more streamlined interface and a revision of the subtraction analysis [40] originally developed by Laird et al. [37] that has been substantially improved using the new statistical framework developed by Eickhoff et al. [38]. Tools are also included to spatially renormalize coordinates to Talairach or MNI space using publicly available algorithms $[21,22]$.

All of the above software applications can be downloaded from the BrainMap website [41]. [See Additional File 1 for the Scribe user manual, Additional File 2 for the Sleuth user manual, and Additional File 3 for the GingerALE user manual]

\section{Related Tools}

In addition to Scribe, Sleuth, and GingerALE, BrainMap distributes or links to several other related tools that serve ancillary functions for carrying out coordinatebased neuroimaging meta-analyses and sharing coordinate-based data and metadata.

1. BrainMapWeb is a web-based application for searching and retrieving data from the functional database [42]. Queries are similar to those of Sleuth, but lack 3D visualizations and advanced data manipulation capabilities.

2. icbm2tal is a coordinate-based transformation that was developed to accommodate spatial disparity between Talairach and MNI coordinates [21]. icbm2tal has been shown to provide improved fit as compared to the earlier mni2tal transform [22], and improve the accuracy of coordinate-based meta-analyses [23]. $i c b m 2 t a l$ is distributed from within GingerALE or can be downloaded as MATLAB .m files [43].

3. Mango (Multi-image Analysis GUI) is a viewer for biomedical research images [44]. It provides analysis tools and a user interface to navigate image volumes. Mango is available as a desktop application, web application, or iPad application. In the context of the BrainMap project, Mango may be used for viewing meta-analysis results and generating and editing ROIs for Sleuth's image-based ROI searches.

4. The Talairach Daemon is a spatially comprehensive set of anatomical labels for Talairach coordinates $[45,46]$. The Talairach Daemon is available as a desktop java client, web applet, or high-speed database server [47]. The Talairach Daemon is utilized by BrainMap to apply anatomical labels to coordinates 
archived in the databases and to label the centers of mass of ALE meta-analysis results in GingerALE.

5. The Anatomy Toolbox is a MATLAB-based software tool [48] that allows the comparison of statistical images, including meta-analysis results, with probabilistic cytoarchitectonic maps of the human brain $[49,50]$. Consistent findings from neuroimaging may be related to the histological properties of the cerebral cortex. In turn, regions of interest defined by cytoarchitectonic areas [51] may be used for probing the BrainMap databases.

6. $\operatorname{CogPO}$ [52] is an ontology of cognitive paradigms that is being built to enable the formal, machine-interpretable representation of paradigms in cognitive neuroscience experiments [53]. $\operatorname{CogPO}$ is based on the BrainMap taxonomy for describing experiments, and utilizes both the BrainMap functional database and the Functional Imaging Biomedical Informatics Research Network Human Imaging Database [54] for development and evaluation. $\operatorname{Cog} P O$ version 1 is available as a wiki [55] or can be downloaded in OWL format [56]. $\operatorname{CogPO}$ also is available from within the Neuroscience Information Framework NeuroLex Wiki [57] and the National Center for Biomedical Ontology BioPortal [58].

\section{Two Exemplar Meta-Analyses}

In this section, we provide two examples of how coordinate-based neuroimaging meta-analyses can be performed using the BrainMap system of databases and software applications.

\section{ALE Meta-Analysis of Acupuncture Studies}

Paradigm-based, or function-based, meta-analyses are analyses in which coordinates are pooled from a set of published neuroimaging studies examining similar behavioral conditions, as a means to determine the most consistently observed activation pattern for a given task. As an example, we performed a paradigm-based meta-analysis to identify consistent results observed during acupuncture tasks in functional neuroimaging studies. A Sleuth search for the experiments reporting activations in healthy subjects was constructed using multiple search criteria: (1) Experiments: Paradigm Class IS "Acupuncture", (2) Experiments: Context IS "Normal Mapping", and (3) Experiments: Activation IS "Activations Only". This Sleuth query returned hits for 10 papers, with 23 experiments; however, the search results were then manually filtered in Sleuth's workspace by toggling experiments to remove activations associated with sham acupuncture. Thus, the final meta-analytic data set included 180 coordinates of brain activation locations from 10 papers across 20 experiments. These coordinates were exported from Sleuth for ALE meta-analysis using GingerALE. GingerALE also accepts text files generated manually by the user (i.e., without Sleuth); however, the formatting must match Sleuth's output. [See Additional File 4 for an example of a BrainMap-formatted text file that can be read by Ginger$A L E]$ Figure 2 depicts the procedure for (a) searching, (b) filtering, and (c) visualizing the acupuncture workspace in Sleuth, and (d) reveals the results of ALE meta-analysis of this data set as viewed in Mango. The strongest convergence of foci from this group of acupuncture studies was observed in the bilateral insula, postcentral gyri, inferior parietal lobule, thalamus, and cerebellum. These regions are generally associated with stimulation of the somatosensory system, and are likely candidates for regions engaged during acupuncture tasks.

\section{Meta-Analytic Connectivity Modeling of the Amygdala}

In contrast to a paradigm-based meta-analysis, we next demonstrate how structure-based meta-analyses are carried out using BrainMap software using the meta-analytic connectivity modeling (MACM) approach. MACM was developed as a method of investigating whole-brain coactivation patterns for a region of interest across a range of tasks, i.e., functional connectivity. In this technique, the BrainMap database is used to search for studies reporting normal mapping activations in healthy subjects that fall within the boundaries of a threedimensional rectangular, spherical, or arbitrary-shaped ROI, regardless of the behavioral conditions employed. The whole brain activation patterns from these studies are then integrated using the ALE method, yielding a map of significant coactivations that provides a task-free meta-analytic model of the region's functional interactions throughout the rest of the brain. This method can be viewed as the meta-analytic analogue to seed-based connectivity analyses of resting state fMRI data [59-61]. MACM analyses have been shown to be useful in understanding the functional connectivity of the amygdala [62], parietal operculum [63], regions of the default mode network [64], and the nucleus accumbens [65].

As an example, we performed a MACM analysis of the left amygdala, using an ROI defined from the HarvardOxford Structural Probability Atlas distributed with the FSL software $[15,66]$ and converted to Talairach space. This ROI was utilized by Robinson et al., although in that study the ROI was thresholded to $70 \%$ probability [62]. A Sleuth search for the experiments reporting activations in healthy subjects was constructed using multiple search criteria: (1) Locations: Talairach Image IS "LeftAmygdala. nii.gz", (2) Experiments: Context IS "Normal Mapping", and (3) Experiments: Activation IS "Activations Only". [See Additional File 5 for an example of a BrainMap-formatted ROI image file of the left amygdala that can be used for image-based ROI searches in Sleuth] This Sleuth query returned hits for 188 papers, with 263 experiments and 3,305 locations matching the search criteria; all of 


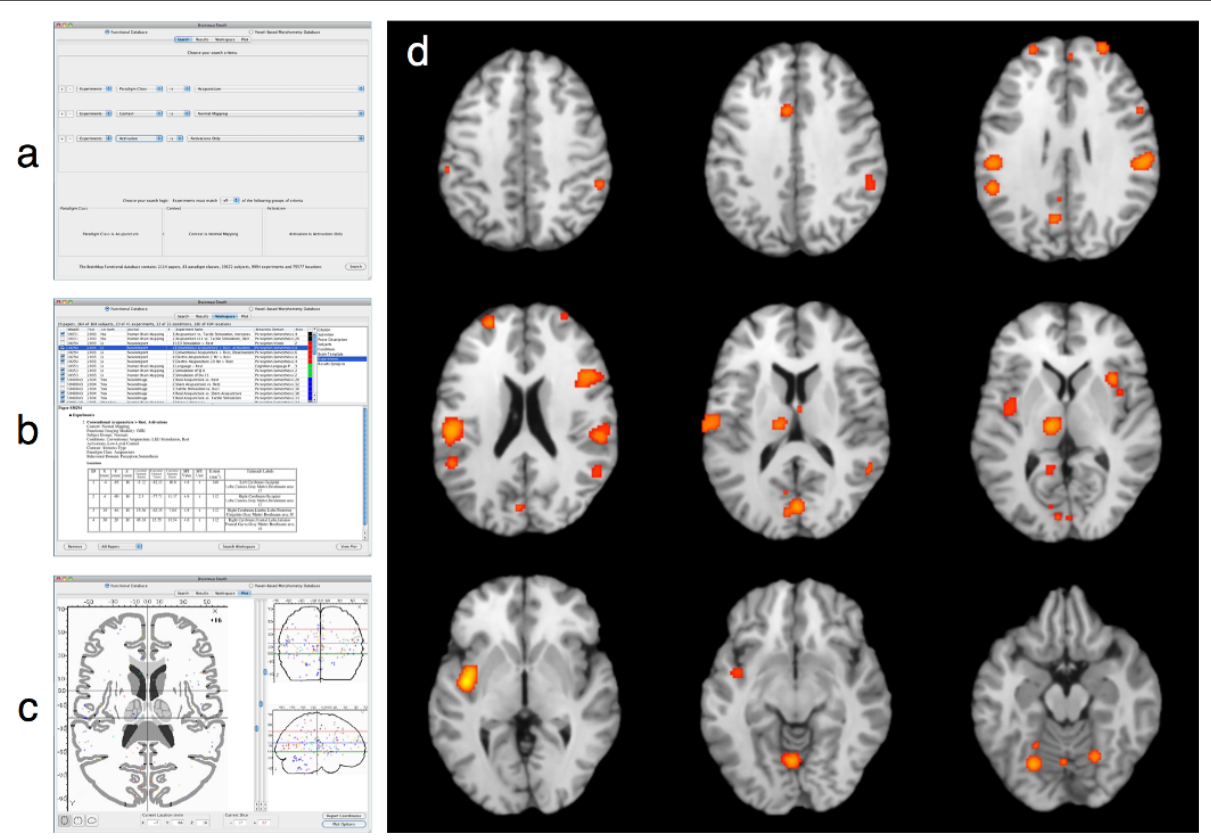

Figure 2 Procedure and Results for a Paradigm-Based ALE Meta-Analysis. In paradigm-based, or function-based, meta-analyses, the BrainMap database is searched for a paradigm or task of interest by (a) constructing an appropriate set of search criteria within Sleuth. Studies matching this query are (b) downloaded to Sleuth's workspace panel for further filtering, and (c) the observed location results of these experiments can be visualized on a glass brain. Using GingerALE, these locations can be meta-analyzed using the ALE approach, and (d) the ALE results can be visualized using Mango.

these coordinates were exported as a text file to be metaanalyzed with GingerALE. Figure 3 depicts (a) the visualization of the left amygdala ROI in Mango, (b) the search criteria in Sleuth, (c) visualization of the left amygdala workspace in Sleuth, and (d) the results of the MACM analysis of this data set in Mango. As reported by Robinson et al. [62], the strongest convergence of foci of left amygdala coactivations was observed in the bilateral amygdala, posterior and anterior cingulate, inferior and medial frontal gyri, insula, thalamus, and fusiform gyri.

\section{Conclusions}

The BrainMap project, including its databases, software clients, and other associated tools, will continue to evolve in response to the meta-analytic needs of biomedical researchers in the structural and functional neuroimaging communities. Current work on BrainMap focuses on further extensions of the analysis capabilities, such as adding conjunction analysis of multiple data sets to GingerALE [67] and the ability to run a cluster analysis on a statistical parametric image. Tools are also being developed to integrate BrainMap metadata in Mango to facilitate detailed and quantitative functional labeling of any given image in the viewer (e.g., a single region of interest or multiple regions from an activation map). In addition, we are currently working on adding capabilities for carrying out statistical testing of Sleuth's metadata histograms to determine the significant behavioral domains or paradigms that have been most frequently reported as corresponding to a given ROI or set of ROIs. In the next year, BrainMapWeb will also undergo a substantial upgrade that will include a more efficient interface and integration of VBM searches. Lastly, we aim to update the hardware that serves the BrainMap database, in order improve the computational speed of image-based ROI searches in Sleuth and reduce the technical limitations that have been imposed, particularly with respect to the size of the ROIs allowed.

The BrainMap project's overall goal is to provide the human brain mapping community with data sets, computational tools, and neuroinformatics resources that enable quantitative meta-analyses and meta-analysis-based neuroimaging data interpretation. Our philosophy is that the most compelling meta-analytic applications are those extend the ALE method beyond that of a purely retrospective tool and utilize meta-analytic results to guide prospective analyses in newly acquired experimental neuroimaging data. Our aim is to fully embrace this philosophy in the next phase of the BrainMap project as we develop novel meta-analytic tools for improving causal model fit when studying the temporal dynamics that are engaged across different brain regions using effective connectivity techniques, such as dynamic causal modeling [68] and structural equation modeling [69]. Similarly, our most recent work emphasizes our meta-analytic philosophy via large-scale data mining as a means to 


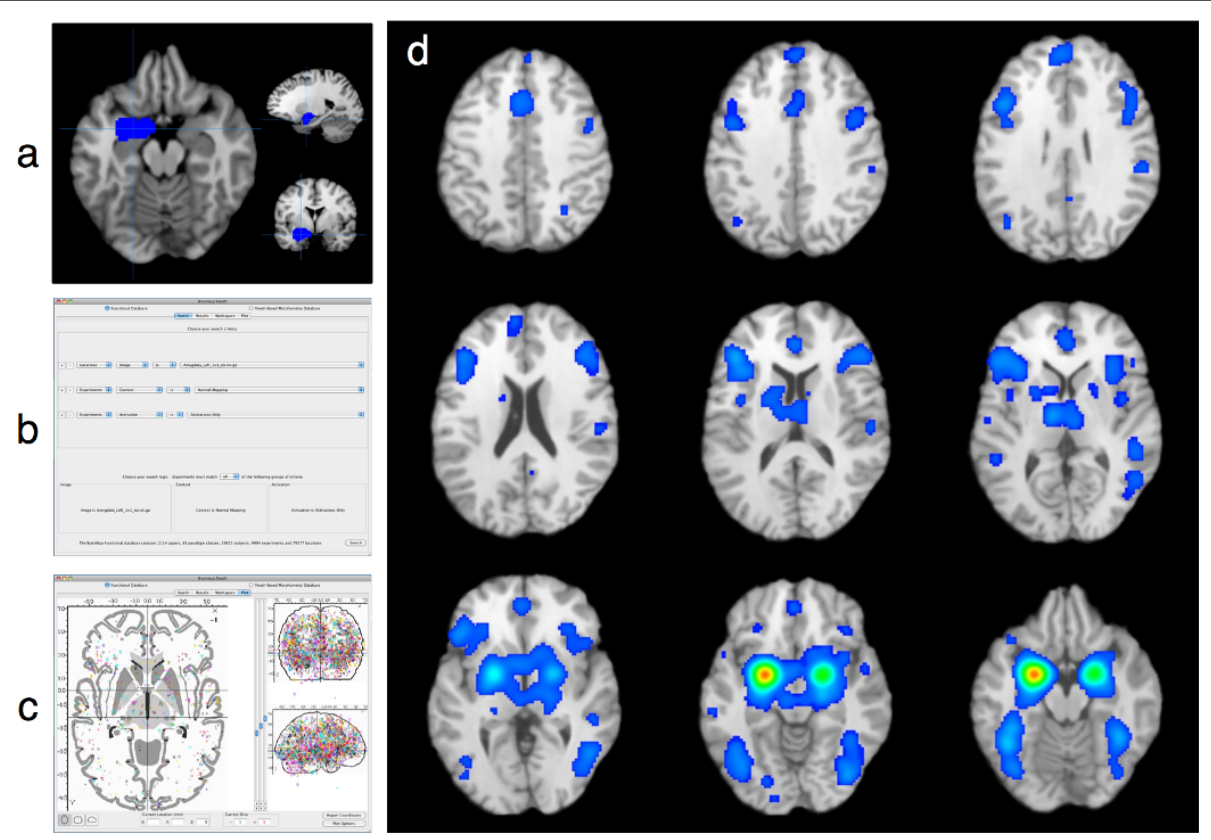

Figure 3 Procedure and Results for a Meta-Analytic Connectivity Modeling Analysis. In meta-analytic connectivity modeling (MACM) analyses, the BrainMap database is searched for activations in healthy subjects that are reported within the boundaries of a three-dimensional rectangular or arbitrary-shaped ROI. To identify the regions that coactivate with this ROI, a user must (a) obtain or generate a gzipped NIfTI image file that identifies the desired region of interest, and (b) construct an appropriate set of search criteria within Sleuth. Studies matching this query are downloaded to Sleuth's workspace and (c) the observed locations reporting across these experiments are visualized on Sleuth's glass brain. After meta-analysis using GingerALE, (d) the MACM results can be visualized in Mango.

investigate fundamental brain-behavior correlations and the organization and interactions within intrinsic connectivity networks [70,71]. Future work on the BrainMap project will additionally involve the development of more comprehensive data mining techniques, as well as extending the functionality of meta-analytic connectivity mapping tools, including constructing, validating, and distributing an atlas of whole-brain task-dependent connectivity. Within the scope of these future aims, the BrainMap project hopes to achieve significant progress in our long-term vision to provide researchers with the tools and data that will provide the foundations for neuroimaging-based models of healthy brain function, as well as models of psychiatric or neurological disease, across the human lifespan.

\section{Additional material}

Additional file 1: BrainMap Scribe Software Manual. This file describes the features of the Scribe desktop application for creating BrainMap database entries.

Additional file 2: BrainMap Sleuth Software Manual. This file describes the features of the Sleuth desktop application for searching, retrieving, and visualizing data archived in the BrainMap databases.

Additional file 3: BrainMap GingerALE Software Manual. This file describes the features of the GingerALE desktop application for performing activation likelihood estimation (ALE) meta-analyses on coordinate-based neuroimaging data.
Additional file 4: BrainMap GingerALE Coordinate File. This file is an example of a BrainMap-formatted text file of coordinates that can be read and analyzed using GingerALE.

Additional file 5: Gzipped NIfTI Image File of the Left Amygdala for Arbitrary-Shaped ROI Search in Sleuth. This file is an example of a BrainMap-formatted image file that can be used for arbitrary-shaped ROI searches in Sleuth.

\section{Acknowledgements}

This work was supported by grants from the National Institute of Mental Health (R01-MH074457 and R01-MH084812) and the Helmholz Initiative on Systems-Biology "The Human Brain Model".

\section{Author details}

${ }^{1}$ Research Imaging Institute, University of Texas Health Science Center, San Antonio, TX, USA. ${ }^{2}$ Department of Psychiatry and Psychotherapy, RWTH Aachen University, Germany. ${ }^{3}$ Institute of Neuroscience and Medicine (INM 2), Research Center Jülich, Jülich, Germany. ${ }^{4}$ Department of Physics and Earth Sciences, St. Mary's University, San Antonio, TX, USA. ${ }^{5}$ Scott \& White Memorial Hospital, Neuroscience Institute, Temple, TX, USA. ${ }^{6}$ Texas A\&M Health Science Center, College of Medicine, Temple, TX, USA. ${ }^{7}$ The Mind Research Network, Albuquerque, NM, USA. ${ }^{8}$ Department of Neurology, University of Pennsylvania, Philadelphia, PA, USA.

\section{Authors' contributions}

$A R L$ and PTF designed the study. ARL wrote the manuscript. SBE, PMF, $A M U$, and $J L L$ developed and managed the software applications, databases, and other resources. SBE, DB, and PET contributed algorithms and tools to the software. KLR, JJS, DRM, JLR, and RWL contributed and analyzed data and tested the software. All authors read and approved the final manuscript. 


\section{Competing interests}

The authors declare that they have no competing interests.

Received: 26 May 2011 Accepted: 9 September 2011

Published: 9 September 2011

\section{References}

1. Hrynaszkiewicz I: A call for BMC Research Notes contributions promoting best practice in data standardization, sharing, and publication. BMC Res Notes 2010, 3:235.

2. The Biomedical Informatics Research Network. [http://nbirn.net].

3. Keator DB, Grethe JS, Marcus D, Ozyurt B, Gadde S, Murphy S, Pieper S, Greve D, Notestine R, Bockholt HJ, Papadopoulos P, BIRN: A national human neuroimaging collaboratory enabled by the Biomedical Informatics Research Network (BIRN). IEEE Trans Inf Technol Biomed 2008, 12:162-72.

4. XNAT Central. [http://central.xnat.org].

5. Marcus DS, Olsen TR, Ramaratnam M, Buckner RL: The Extensible Neuroimaging Archive Toolkit: An informatics platform for managing, exploring, and sharing neuroimaging data. Neuroinformatics 2007, 5:11-34.

6. The Alzheimer's Disease Neuroimaging Initiative. [http://www.adni-info. org].

7. Butcher J: Alzheimer's researchers open the doors to data sharing. The Lancet Neurology 2007, 6:480-481.

8. The Human Connectome Project. [http://humanconnectome.org].

9. Akil H, Martone ME, Van Essen DC: Challenges and opportunities in mining neuroscience data. Science 2011, 331:708-712.

10. Mackenzie-Graham AJ, Van Horn JD, Woods RP, Crawford KL, Toga AW: Provenance in neuroimaging. Neurolmage 2008, 42:178-195.

11. Fox PT, Lancaster JL: Mapping context and content: The BrainMap model. Nat Rev Neurosci 2002, 3:319-321.

12. Laird AR, Lancaster JL, Fox PT: BrainMap: The social evolution of a human brain mapping database. Neuroinformatics 2005, 3:65-78.

13. Laird AR, Lancaster JL, Fox PT: Lost in localization? The focus is metaanalysis. Neuroimage 2009, 48:18-20.

14. Laird AR, Eickhoff SB, Kurth F, Fox PM, Uecker AM, Turner JA, Robinson JL, Lancaster JL, Fox PT: ALE meta-analysis workflows via the BrainMap database: Progress towards a probabilistic functional brain atlas. Front Neuroinformatics 2009, 3:23.

15. FMRIB Software Library. [http://www.fmrib.ox.ac.uk/fsl].

16. Statistical Parametric Mapping. [http://www.fil.ion.ucl.ac.uk/spm].

17. Analysis of Functional Neurolmages. [http://afni.nimh.nih.gov/afni].

18. Fox PT: Spatial normalization origins: Objectives, applications, and alternatives. Hum Brain Mapping 1995, 3:161-164.

19. Talairach J, Tournoux P: Co-planar stereotaxic atlas of the human brain: 3dimensional proportional system: An approach to cerebral imaging. Stuttgart: Georg Thieme 1988.

20. Collins DL, Neelin P, Peters TM, Evans AC: Automatic 3D intersubject registration of MR volumetric data in standardized Talairach space. $J$ Comput Assist Tomogr 1994, 18:192-205.

21. Lancaster JL, Tordesillas-Gutiérrez D, Martinez M, Salinas F, Evans A, Zilles $K$, Mazziotta JC, Fox PT: Bias between MNI and Talairach coordinates analyzed using the ICBM-152 brain template. Hum Brain Mapp 2007, 28:1194-1205.

22. Brett M, Johnsrude IS, Owen AM: The problem of functional localization in the human brain. Nat Rev Neurosci 2002, 3:243-249.

23. Laird AR, Robinson JL, McMillan KM, Tordesillas-Gutiérrez D, Moran ST, Gonzales SM, Ray KL, Franklin C, Glahn DC, Fox PT, Lancaster JL: Comparison of the disparity between Talairach and MNI coordinates in functional neuroimaging data: validation of the Lancaster transform. Neurolmage 2010, 51:677-683.

24. fMRI Methods Working Group. [http://www.fmrimethods.org].

25. Fox PT, Lancaster JL: Neuroscience on the net. Science 1994, 266:994-996.

26. BrainMap Publications (1994-Present). [http://brainmap.org/pubs].

27. Derrfuss J, Mar RA: Lost in localization: The need for a universal coordinate database. Neurolmage 2009, 48:1-7.

28. Glahn DC, Laird AR, Ellison-Wright I, Thelen SM, Robinson JL, Lancaster JL, Bullmore E, Fox PT: Meta-analysis of gray matter anomalies in schizophrenia: Application of anatomic likelihood estimation and network analysis. Biol Psychiatry 2008, 64:774-781.
29. Ellison-Wright I, Glahn DC, Laird AR, Thelen SM, Bullmore ET: The anatomy of first-episode and chronic schizophrenia: An anatomical likelihood estimation meta-analysis. Am J Psych 2008, 165:1015-1023.

30. Nickl-Jockschat T, Habel U, Michel TM, Manning J, Laird A, Fox PT, Schneider F, Eickhoff SB: Brain structure anomalies in autism spectrum disorder (ASD): A meta-analysis of VBM studies using anatomic likelihood estimation (ALE). Hum Brain Mapp

31. Oracle Corporation. [http://www.oracle.com].

32. Fox PT, Laird AR, Fox SP, Fox PM, Uecker AM, Crank M, Koenig SF, Lancaster JL: BrainMap taxonomy of experimental design: Description and evaluation. Hum Brain Mapp 2005, 25:185-198.

33. Apple iTunes. [http://www.apple.com/itunes].

34. The Neuroimaging Informatics Technology Initiative. [http://nifti.nimh.nih. gov].

35. Thomson Reuters EndNote. [http://endnote.com].

36. Turkeltaub PE, Eden GF, Jones KM, Zeffiro TA: Meta-analysis of the functional neuroanatomy of single-word reading: Method and validation. Neurolmage 2002, 16:765-780.

37. Laird AR, Fox M, Price CJ, Glahn DC, Uecker AM, Lancaster JL, Turkeltaub PE, Kochunov P, Fox PT: ALE meta-analysis: Controlling the false discovery rate and performing statistical contrasts. Hum Brain Mapp 2005, 25:155-164.

38. Eickhoff SB, Laird AR, Grefkes C, Wang LE, Zilles K, Fox PT: Coordinatebased activation likelihood estimation meta-analysis of neuroimaging data: A random-effects approach based on empirical estimates of spatial uncertainty. Hum Brain Mapp 2009, 30:2907-2926.

39. Turkeltaub PE, Eickhoff SB, Laird AR, Fox M, Wiener M, Fox P: Minimizing within-experiment and within-group effects in activation likelihood estimation meta-analyses. Hum Brain Mapp .

40. Eickhoff SB, Bzdok D, Laird AR, Roski C, Caspers S, Zilles K, Fox PT: Coactivation patterns distinguish cortical modules, their connectivity and functional differentiation. Neuroimage.

41. BrainMap Project. [http://www.brainmap.org].

42. BrainMapWeb [http://www.brainmap.org:9000/bmapWeb].

43. The icbm2tal Transform. [http://www.brainmap.org/icbm2tal].

44. Mango [http://ric.uthscsa.edu/mango].

45. Lancaster JL, Rainey LH, Summerlin JL, Freitas CS, Fox PT, Evans AC, Toga AW, Mazziotta JC: Automated labeling of the human brain: A preliminary report on the development and evaluation of a forwardtransform method. Hum Brain Mapp 1997, 5:238-242.

46. Lancaster JL, Woldorff MG, Parsons LM, Liotti M, Freitas CS, Rainey L, Kochunov PV, Nickerson D, Mikiten SA, Fox PT: Automated Talairach Atlas labels for functional brain mapping. Hum Brain Mapp 2000, 10:120-131.

47. The Talairach Daemon. [http://www.talairach.org].

48. The Anatomy Toolbox. [http://www.fz-juelich.de/inm/inm-1/DE/Forschung/ _docs/SPMAnantomyToolbox/SPMAnantomyToolbox_node.html].

49. Eickhoff S, Stephan KE, Mohlberg H, Grefkes C, Fink GR, Amunts K, Zilles K: A new SPM toolbox for combining probabilistic cytoarchitectonic maps and functional imaging data. Neurolmage 2005, 25:1325-1335.

50. Eickhoff SB, Paus T, Caspers S, Grosbras MH, Evans A, Zilles K, Amunts K: Assignment of functional activations to probabilistic cytoarchitectonic areas revisited. Neurolmage 2007, 36:511-521.

51. Eickhoff SB, Heim S, Zilles K, Amunts K: Testing anatomically specified hypotheses in functional imaging using cytoarchitectonic maps. Neurolmage 2006, 32:570-582.

52. The Cognitive Paradigm Ontology. [http://www.cogpo.org].

53. Turner JA, Laird AR: The Cognitive Paradigm Ontology: Design and application. Neuroinformatics

54. Keator DB, Wei D, Gadde S, Bockholt J, Grethe JS, Marcus D, Aucoin N, Ozyurt IB: Derived data storage and exchange workflow for large-scale neuroimaging analyses on the BIRN Grid. Front Neuroinformatics 2009, 8:231-249.

55. The CogPO Wiki. [http://www.wiki.cogpo.org]

56. The CogPO, Version 1. [http://www.cogpo.org/ontologies/CogPOver1.owl].

57. The Neuroscience Information Framework NeuroLex Wiki. [http://neurolex org/wiki].

58. The National Center for Biomedical Ontology. BioPortal [http://www. bioportal.ncbo.org].

59. Biswal B, Yetkin FZ, Haughton VM, Hyde JS: Functional connectivity in the motor cortex of resting human brain using echo-planar MRI. Magn Reson Med 1995, 34:537-541. 
60. Xiong J, Parsons LM, Gao JH, Fox PT: Interregional connectivity to primary motor cortex revealed using MRI resting state images. Hum Brain Mapp 1999, 8:151-156.

61. Cordes D, Haughton VM, Arfanakis K, Wendt GJ, Turski PA, Moritz CH, Quigley MA, Meyerand ME: Mapping functionally related regions of brain with functional connectivity MR imaging. AJNR Am J Neuroradiol 2000, 21:1636-1644

62. Robinson JL, Laird AR, Glahn DC, Lovallo WR, Fox PT: Meta-analytic connectivity modelling: Delineating the functional connectivity of the human amygdala. Hum Brain Mapp 2010, 31:173-184.

63. Eickhoff S, Jbabdi S, Caspers S, Laird AR, Fox PT, Zilles K, Behrens T: Anatomical and functional connectivity of cytoarchitectonic areas within the human parietal operculum. J Neurosci 2010, 30:6409-6421.

64. Laird AR, Eickhoff SB, Li K, Robin DA, Glahn DC, Fox PT: Investigating the functional heterogeneity of the default mode network using coordinatebased meta-analytic modeling. J Neurosci 2009, 29:14496-14505.

65. Cauda F, Cavanna AE, D'agata F, Sacco K, Duca S, Geminiani GC: Functional connectivity and coactivation of the nucleus accumbens: A combined functional connectivity and structure-based meta-analysis. $J$ Cogn Neurosci.

66. Smith SM, Jenkinson M, Woolrich MW, Beckmann CF, Behrens TE, JohansenBerg H, Bannister PR, De Luca M, Drobnjak I, Flitney DE, Niazy RK, Saunders J, Vickers J, Zhang Y, De Stefano N, Brady JM, Matthews PM: Advances in functional and structural MR image analysis and implementation as FSL. Neuroimage 2004, 23:S208-S219.

67. Caspers S, Zilles K, Laird AR, Eickhoff SB: ALE meta-analysis of action observation and imitation in the human brain. Neuroimage 2010, 50:1148-1167.

68. Friston $\mathrm{K}$, Harrison L, Penny W: Dynamic causal modelling. Neuroimage 2003, 19:1273-1302.

69. McIntosh A, Gonzales-Lima F: Structural equation modeling and its application to network analysis in functional brain imaging. Hum Brain Mapp 1994, 2:2-22.

70. Smith SM, Fox PT, Miller KL, Glahn DC, Fox PM, Mackay CE, Filippini N, Watkins KE, Toro R, Laird AR, Beckmann CF: Correspondence of the brain's functional architecture during activation and rest. Proc Natl Acad Sci USA 2009, 106:13040-13045.

71. Laird AR, Fox PM, Eickhoff SB, Turner JA, Ray KL, McKay DR, Glahn DC, Beckmann CF, Smith SM, Fox PT: Behavioral interpretations of intrinsic connectivity networks. J Cogn Neurosci .

doi:10.1186/1756-0500-4-349

Cite this article as: Laird et al:: The BrainMap strategy for

standardization, sharing, and meta-analysis of neuroimaging data. BMC

Research Notes 2011 4:349.

\section{Submit your next manuscript to BioMed Central and take full advantage of:}

- Convenient online submission

- Thorough peer review

- No space constraints or color figure charges

- Immediate publication on acceptance

- Inclusion in PubMed, CAS, Scopus and Google Scholar

- Research which is freely available for redistribution

Submit your manuscript at www.biomedcentral.com/submit 